DOI: $10.23857 /$ dc.v5i1.927

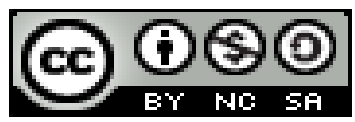

Ciencias de la salud

Artículo de investigación

\title{
Estudio de casos sobre la cirrosis hepática y sus complicaciones en el Hospital Universitario de Guayaquil, año 2015
}

\section{Case study on cirrhosis of the liver and its complications at the University Hospital of Guayaquil, 2015}

\section{Estudo de caso sobre cirrose hepática e suas complicações no Hospital Universitário de Guayaquil, 2015}

\author{
María Fernanda Delgado-López ${ }^{\text {I }}$ \\ mayferdelo@hotmail.com \\ Jonathan Yomar Chamaidan-Moreno II \\ jonayo85@hotmail.com
}

Karen Lisseth Labanda-Jaramillo ${ }^{\text {III }}$

dra.karenlabanda@hotmail.com
Diana Patricia Delgado-Suárez ${ }^{\text {IV }}$
patico_ds89@hotmail.com

Diana Gabriela Echeverría-Gia ${ }^{\mathrm{V}}$ gabyechvrria@gmail.com

Stefany Gladyz Matute-Ortiz VI stefy_16_05@hotmail.com

Recibido: 23 de octubre de 2018 *Corregido: 25 de noviembre de 2018 * Aceptado: 10 de diciembre de 2018

I. Médico, Instituto Ecuatoriano de Seguridad Social (IESS), Machala, Ecuador.

II. Médico, Instituto Ecuatoriano de Seguridad Social (IESS), Machala, Ecuador.

III. Médico, Instituto Ecuatoriano de Seguridad Social (IESS), Machala, Ecuador.

iv. Médico, Clínica Virgen de Monserrate, Montecristi, Ecuador.

v. Médico, Instituto Ecuatoriano de Seguridad Social (IESS), Machala, Ecuador.

v. Médico, Instituto Ecuatoriano de Seguridad Social (IESS), Machala, Ecuador. 


\section{Resumen}

La Cirrosis Hepática es una de las enfermedades más comunes dentro del Hospital Universitario de Guayaquil. Durante el período 2015 se han registrado aproximadamente 1.050 casos en las diferentes edades, tomando como muestra 275 pacientes registrado durante el período enero hasta diciembre del 2015 en el área de emergencia del mismo. El propósito de este proyecto de investigación es enfocarnos en la enfermedad, sus complicaciones y los factores de riego que se asocian a los pacientes que acuden en busca de la emergencia del Hospital, poder verificar además cual es la complicación más común por la que los pacientes terminan hospitalizados. Tomando en consideración alrededor del $31 \%$ corresponde a Ascitis, 63\% Hemorragia Digestiva, 4\% Síndrome Hepatorrenal y Varices esofágicas $6 \%$ en forma general. Para llevar a cabo esta investigación se tomó el modelo de investigación descriptiva de campo, Hernández, Fernández y Baptista (2014), con el método de estudio de casos y el análisis de contenido, la estadística descriptiva revisando la información de datos estadísticos de historias clínicas de los 275 pacientes. Gracias a ello, también pudimos observar dentro de nuestro estudio que los factores de riesgo más comunes son: el alcoholismo, la elevación de triglicéridos en sangre (hipercolesterolemia familiar), el antecedente de enfermedad por hepatitis b, entre otros. Las Complicaciones de la Enfermedad Hepática actualmente son muy frecuentes en nuestro medio por diferentes factores de riesgo. Ello puede variar dependiendo del sexo, la edad, calidad de vida, el consumo de alcohol, consumos prolongados de medicamentos tóxicos, inmunológicos, genéticos y metabólicos. De acuerdo con la edad se evidencia que, la etapa más susceptible se ubica entre el rango de 60-65 años de edad con un porcentaje del 64\%, mientras que en el sexo el género más afectado con hemorragia digestiva es el masculino con un porcentaje del 69,08\%. Dentro de los factores de riesgo el alcohol ocupada el 60\%.

Palabras Clave: Cirrosis hepática; complicaciones; estudio de caso; hospital universitario

\section{Abstract}

Hepatic Cirrhosis is one of the most common diseases within the University Hospital of Guayaquil. During the 2015 period approximately 1,050 cases were registered in the different ages, taking as a sample 275 patients registered during the period January 2015 to December 2015 in the emergency area of the same. The purpose of this research project is to focus on the disease, its complications and

760 Vol. 5, núm. 3, enero, 2019, pp.759-779

María Fernanda Delgado López, Jonathan Yomar Chamaidan Moreno, Karen Lisseth Labanda Jaramillo, Diana Patricia Delgado Suárez, Diana Gabriela Echeverría Gia, Stefany Gladyz Matute Ortiz 
the risk factors that are associated with patients who come in search of the emergency of the Hospital, to be able to verify also which is the most common complication for which the patients Patients end up hospitalized. Taking into consideration about 31\% corresponds to Ascitis, 63\% Digestive Hemorrhage, 4\% Hepatorenal Syndrome and 6\% esophageal varices in general. To carry out this research, the field descriptive research model, Hernández, Fernández and Baptista (2014), was taken with the case study method and the content analysis, the descriptive statistics reviewing the information from statistical data of medical records of the 275 patients. Thanks to this, we could also observe in our study that the most common risk factors are: alcoholism, elevated blood triglycerides (familial hypercholesterolemia), and the antecedent of hepatitis b disease, among others. Complications of Liver Disease are currently very frequent in our environment due to different risk factors. This may vary depending on sex, age, quality of life, and alcohol consumption, prolonged consumption of toxic, immunological, genetic and metabolic drugs. According to age, it is evident that the most susceptible age is between the ranges of 60-65 years of age with a percentage of $64 \%$, while in sex the most affected gender with digestive hemorrhage is the male with a percentage of $69.08 \%$. Within the risk factors alcohol occupied 60\%.

Palabras Clave: Liver cirrhosis; complications; case study; University Hospital

Key words: Lung disease; quality of life; patients; University Hospital.

\section{Resumo}

A cirrose hepática é uma das doenças mais comuns no Hospital Universitário de Guayaquil. Durante o período de 2015 foram registrados aproximadamente 1.050 casos nas diferentes idades, tendo como amostra 275 pacientes cadastrados no período de janeiro de 2015 a dezembro de 2015 na área de emergência do mesmo. O objetivo deste projeto de pesquisa é enfocar a doença, suas complicações e os fatores de risco associados aos pacientes que vêm em busca da emergência do Hospital, para verificar também qual a complicação mais comum pela qual os pacientes Os pacientes acabam hospitalizados. Levando-se em consideração, cerca de 31\% corresponde à Ascite, 63\% à Hemorragia Digestiva, $4 \%$ à Síndrome Hepatorrenal e $6 \%$ às varizes esofágicas em geral. Para realizar esta pesquisa, utilizou-se o modelo de pesquisa descritiva de campo, Hernández, Fernández e Baptista (2014), com o método de estudo de caso e a análise de conteúdo, a estatística descritiva revisando as

761 Vol. 5, núm. 3, enero, 2019, pp.759-779

María Fernanda Delgado López, Jonathan Yomar Chamaidan Moreno, Karen Lisseth Labanda Jaramillo, Diana Patricia Delgado Suárez, Diana Gabriela Echeverría Gia, Stefany Gladyz Matute Ortiz 
informações dos dados estatísticos dos prontuários médicos, dos 275 pacientes. Graças a isso, também pudemos observar em nosso estudo que os fatores de risco mais comuns são: alcoolismo, triglicérides sanguíneos elevados (hipercolesterolemia familiar), antecedente de hepatite b, entre outros. As complicações da doença hepática são atualmente muito frequentes em nosso meio devido a diferentes fatores de risco. Isso pode variar dependendo do sexo, idade, qualidade de vida, consumo de álcool, consumo prolongado de drogas tóxicas, imunológicas, genéticas e metabólicas. De acordo com a idade, é evidente que a faixa etária mais suscetível está na faixa de 60-65 anos com um percentual de $64 \%$, enquanto no sexo o gênero mais afetado com hemorragia digestiva é o masculino com percentual de $69,08 \%$. Dentro dos fatores de risco, o álcool ocupou $60 \%$.

Palabras Clave: cirrose hepática; complicações; estudo de caso; hospital Universitário.

\section{Introducción}

La Cirrosis Hepática es el estadio final de todas las enfermedades hepáticas crónicas progresivas. Es un proceso difuso caracterizado por la pérdida de parénquima hepático, formación de septos fibrosos y de nódulos de regeneración que causan la distorsión de la arquitectura y anatomía vascular normal, aproximadamente, el 40-60\% de los casos en Europa y Norteamérica son debidos al abuso de alcohol y al hígado graso no alcohólico, mientras que el $25-30 \%$ es el resultado de la hepatitis crónica vírica. Actualmente se considera que la cirrosis es una enfermedad dinámica y potencialmente reversible en estadios iniciales. (Buey, 2012).

Se estima que la cirrosis se halla detrás de 800.000 muertes anuales en todo el mundo. En Europa y Estados Unidos tiene una prevalencia de alrededor de 250 casos anuales por cada 100.000 personas. En los varones la prevalencia es dos veces mayor que en las mujeres. (AYALA, 2012). Aunque en los estadios iniciales el paciente puede estar asintomático, la historia natural muestra un progresivo desarrollo de ictericia, hipoalbuminemia, plaquetopenia, alteración del tiempo de protrombina y ascitis. Muchos pacientes sufren hemorragias gastrointestinales recurrentes por varices esofágicas. En los estadios avanzados, destacan los cambios en el estado mental y, en concreto, la encefalopatía hepática.

Hay dos fases, la cirrosis compensada y la descompensada, cada una de ellas con pronóstico distinto y diferente supervivencia. La ascitis es la complicación más frecuente, cerca del $60 \%$ de los pacientes

762 Vol. 5, núm. 3, enero, 2019, pp.759-779

María Fernanda Delgado López, Jonathan Yomar Chamaidan Moreno, Karen Lisseth Labanda Jaramillo, Diana Patricia Delgado Suárez, Diana Gabriela Echeverría Gia, Stefany Gladyz Matute Ortiz 
con cirrosis compensada acaban desarrollando ascitis en los siguientes 10 años. Los pacientes con cirrosis descompensada desarrollan una variedad de síntomas como fatiga, extenuación, falta de apetito, náuseas, ictericia, pérdida de peso, dolor de estómago, impotencia, hematomas y hemorragias y otros problemas potencialmente mortales. (Buey, 2012).

En este trabajo estudiamos los casos con este diagnóstico, la incidencia en la calidad de vida de los pacientes y más aún la incidencia de los factores de riesgo en el padecimiento de esta enfermedad en correspondencia con edad, sexo, hábitos y estilos de vida, entre otros. El propósito de este proyecto de investigación es enfocarnos en los factores de riego que se asocian a los pacientes que acuden en busca de la emergencia del Hospital, poder verificar además cual es la complicación más común por la que los pacientes terminan hospitalizados. Tomando en consideración alrededor del $31 \%$ corresponde a Ascitis, 63\% Hemorragia Digestiva, 4\% Síndrome Hepatorrenal y Varices esofágicas $6 \%$ en forma general.

Para llevar a cabo esta investigación se tomó el modelo de investigación descriptiva de campo, con el método de estudio de casos y análisis de contenido (Hernández, Fernández y Baptista, 2014), revisando la información de datos estadísticos de historias clínicas de los 275 pacientes más sus antecedentes familiares. Gracias a ello también pudimos observar dentro de nuestro estudio descriptivo que los factores de riesgo más comunes son: el alcoholismo, la elevación de triglicéridos en sangre (hipercolesterolemia familiar), el antecedente de enfermedad por hepatitis b, entre otros.

Las Complicaciones de la Enfermedad Hepática actualmente son muy frecuentes en nuestro medio por diferentes factores de riesgo como se refirió anteriormente. Ello puede variar dependiendo del sexo, la edad, calidad de vida, del consumo de alcohol, consumos prolongados de medicamentos tóxicos, inmunológicos, genéticos y metabólicos. De acuerdo con la edad se evidencia que, el período más susceptible se ubica entre el rango de 60-65 años de edad con un porcentaje del 64\%, mientras que en el sexo el género más afectado con hemorragia digestiva es el masculino con un porcentaje del $69,08 \%$. Dentro de los factores de riesgo el alcohol ocupada el 60\%.

\section{Materiales y Métodos}

\section{Caracterización esencial de la enfermedad Cirrosis Hepática}

763 Vol. 5, núm. 3, enero, 2019, pp.759-779

María Fernanda Delgado López, Jonathan Yomar Chamaidan Moreno, Karen Lisseth Labanda Jaramillo, Diana Patricia Delgado Suárez, Diana Gabriela Echeverría Gia, Stefany Gladyz Matute Ortiz 
La cirrosis hepática constituye uno de los principales problemas de salud en el mundo, debido a su alta morbilidad y mortalidad. Las tasas de defunción más elevadas se registran en Moldavia y Hungría, mientras que las cifras más bajas, entre 3 y 5 por 100.000 habitantes, corresponden a Irlanda, Colombia, Holanda, Singapur, Israel y Noruega. En algunos países de América Latina, como Chile y México, la cirrosis hepática ocupa, entre el $5^{\circ}$ y $6^{\circ}$ lugar como causa de muerte general. (FARFAN G, 2002)

La cirrosis hepática es una enfermedad frecuente en el mundo, y su prevalencia varia de un país a otro dependiendo de los factores etiológicos. La cirrosis suele manifestarse hacia la cuarta o quinta década de la vida, aunque hay casos juveniles e incluso infantiles, y no es excepcional que un paciente sea portador de una cirrosis durante muchos años, y ésta se manifieste en la senectud. La cirrosis es una enfermedad muy frecuente en el sexo masculino, probablemente porque la infección por los virus de las hepatitis y el etilismo son más frecuentes en el varón. La raza negra, el hábitat urbano y el menor nivel económico parecen ser factores significativos de riesgo del desarrollo de cirrosis.

En la actualidad, la mala alimentación se considera uno de los principales factores de riesgo que aumentan la incidencia de los casos de cirrosis hepática y, que está relacionado con la falta de conciencia de una buena nutrición, que conlleva a desarrollar enfermedades que predisponen a la aparición precoz de la enfermedad.

Esta investigación se enfoca directamente en informar a los pacientes del Hospital Universitario de Guayaquil sobre cada uno de los factores de riesgo, que no solo ocurre por alcoholismo aun siendo el más importante, también los desórdenes alimenticios son factores de riego a considerar, sino que también se incluyen víricas autoinmunes, metabólicas y procesos obstructivos, entre otras; y dar a conocer las complicaciones intra y extra hepáticas. Cabe recalcar que este proyecto incentiva a promover medidas de prevención e ir de la mano con asesoría nutricional, para así mejorar el estilo de vida y reducir la incidencia, de esta manera se logrará aumentar el grado de supervivencia, y evitar pacientes con complicaciones futuras como hemorragia digestiva, ascitis, encefalopatía hepática, hepatocarcinoma, entre otras.

La cirrosis hepática es el estadio final de todas las enfermedades hepáticas crónicas progresivas. Es una alteración histopatológica difusa del hígado caracterizada por pérdida del parénquima hepático,

764 Vol. 5, núm. 3, enero, 2019, pp.759-779

María Fernanda Delgado López, Jonathan Yomar Chamaidan Moreno, Karen Lisseth Labanda Jaramillo, Diana Patricia Delgado Suárez, Diana Gabriela Echeverría Gia, Stefany Gladyz Matute Ortiz 
formación de septos fibrosos y nódulos de regeneración estructuralmente anormales, dando lugar a una distorsión de la arquitectura hepática normal y a una alteración de la anatomía de la vascularización hepática y de la microcirculación. (Buey, 2012).

\section{Criterios para definir la cirrosis hepática según expertos de la OMS}

En 1977, expertos de la Organización Mundial de la Salud, partiendo de los datos morfológicos consideraron los siguientes criterios para definir la cirrosis:

a. que el proceso sea difuso para excluir lesiones locales o focales

b. que exista necrosis, con lo que queda excluida la fibrosis hepática congénita

c. debe existir regeneración nodular y fibrosis difusa, quedando excluida la hiperplasia nodular regenerativa

d. debe haber distorsión del patrón arquitectural y alteración vascular. Estos dos últimos criterios son importantes porque constituyen la base del diagnóstico histopatológico en las biopsias, y porque motivan en gran medida la fisiopatología de la enfermedad. (Buey, 2012).

$\mathrm{Su}$ historia natural se caracteriza por una fase asintomática, denominada cirrosis compensada, de duración variable. En esta fase los pacientes pueden llegar a tener hipertensión portal y varices esofágicas. A medida que la enfermedad progresa con el desarrollo de mayor hipertensión portal y mayor deterioro de la función hepática aparecen las complicaciones de la denominada cirrosis descompensada como ascitis, hemorragia por varices, encefalopatía hepática o ictericia. El desarrollo de cualquiera de estas complicaciones marca la transición de la fase compensada a la descompensada. La progresión de la enfermedad parece que se acelera, con riesgo de muerte con el desarrollo de complicaciones tales como el de sangrado, insuficiencia renal, ascitis refractaria, síndrome hepatorrenal, síndrome hepatopulmonar y peritonitis bacteriana espontánea. El desarrollo de hepatocarcinoma puede acelerar el curso de la enfermedad tanto en la fase compensada como descompensada. (D'Amico G, 2006)

\section{Historia Natural de la Cirrosis Hepática}

Recientemente, se ha propuesto un nuevo esquema en la historia natural de la cirrosis que describe cuatro fases con unas características clínicas y un pronóstico bien diferenciados:

- Estadio 1: ausencia de varices esofágicas y de ascitis: $1 \%$ de mortalidad al año.

765 Vol. 5, núm. 3, enero, 2019, pp.759-779

María Fernanda Delgado López, Jonathan Yomar Chamaidan Moreno, Karen Lisseth Labanda Jaramillo, Diana Patricia Delgado Suárez, Diana Gabriela Echeverría Gia, Stefany Gladyz Matute Ortiz 
- Estadio 2: varices esofágicas sin antecedente de hemorragia y sin ascitis: 3,4\% de mortalidad al año.

- Estadio 3: presencia de ascitis con o sin varices esofágicas: $20 \%$ de mortalidad al año.

- Estadio 4: hemorragia gastrointestinal por hipertensión portal, con o sin ascitis: $57 \%$ de mortalidad al año. Los estadios 1 y 2 corresponden a pacientes con la fase de cirrosis hepática compensada mientras que los estadios 3 y 4 corresponden con la fase de cirrosis hepática descompensada. (R., 2009)

\section{Algunas de las complicaciones de la cirrosis hepática}

\section{Hipertensión portal}

El desarrollo de hipertensión portal es la complicación más frecuente de la cirrosis hepática. Sus consecuencias: hemorragia por rotura de varices esofágicas, ascitis y trastornos de la función renal, peritonitis bacteriana espontánea, gastropatía y colopatía de la HTP, encefalopatía hepática, síndrome hepatopulmonar y síndrome hepatorrenal, entre otras, representan la principal causa de muerte y de trasplante hepático en los pacientes cirróticos.

La hipertensión portal se define como un incremento del gradiente de presión portal: 1-5 mmHg, que viene determinado por la interrelación entre dos factores el flujo sanguíneo y la resistencia vascular que se enfrenta este flujo. Se caracteriza por la existencia de un aumento patológico de la presión hidrostática en el territorio venoso portal. Este aumento sostenido de la presión portal provoca la formación de una extensa red de vasos colaterales que intentan descomprimir el sistema, derivando parte del flujo sanguíneo portal a la circulación sistémica sin pasar por el hígado. De estas colaterales, las varices esofágicas tienen especial relevancia. (Catalina-Rodríguez, 2012)

El incremento de la RVIH es el mecanismo patogénico inicial de la HTP, que se mantiene y agrava por el aumento del flujo sanguíneo esplácnico, secundario a vasodilatación arteriolar. Inicialmente se consideró que el incremento de la RVIH era irreversible, ocasionado por cambios estructurales en la arquitectura hepática, ligado a la distorsión de la arquitectura vascular originada por la fibrosis, los nódulos de regeneración y fenómenos de microtrombosis u oclusión vascular. En la actualidad, se conoce que este aumento no sólo se debe a lo anteriormente citado, sino también a un componente dinámico debido al aumento reversible del tono sinusoidal, con el resultado de un desequilibrio entre

766 Vol. 5, núm. 3, enero, 2019, pp.759-779

María Fernanda Delgado López, Jonathan Yomar Chamaidan Moreno, Karen Lisseth Labanda Jaramillo, Diana Patricia Delgado Suárez, Diana Gabriela Echeverría Gia, Stefany Gladyz Matute Ortiz 
los estímulos vasoconstrictores y vasodilatadores, que es el responsable del $20-30 \%$ del incremento de la RVIH. Las estructuras responsables de dicho componente dinámico son los miofibroblastos portales y septales, las células estrelladas y las vénulas portales. La activación de estos elementos contráctiles se ve favorecida por un estado de disfunción endotelial en las sinusoides, que da lugar a una insuficiente producción de factores vasodilatadores endógenos, como el óxido nítrico (ON), junto con el aumento de factores vasoconstrictores, como el tromboxano A2, la endotelina y el sistema renina angiotensina.

El conocimiento de estos mecanismos resulta de gran importancia, dado que el componente dinámico de la resistencia vascular intrahepática es reversible y, por tanto, una diana terapéutica. Por otra parte, el shunt portosistémico permite que bacterias o productos bacterianos de procedencia intestinal alcancen la circulación general, lo que favorece la liberación de citocinas que promueven tanto la síntesis de óxido nítrico (ON), como de prostaciclina (PGI2) en la circulación extrahepática, donde provocan una intensa vasodilatación y atenúan la respuesta a estímulos vasoconstrictores.

Finalmente, a través del shunt circulan péptidos gastrointestinales con poder vasoactivo (por ejemplo, glucagón) que escapan al catabolismo hepático ejerciendo efectos vasodilatadores sistémicos. Éstos y otros mecanismos vinculados a la intervención de péptidos vasodilatadores se conjugan para inducir un estado de vasodilatación esplácnica. Este fenómeno contribuye a explicar la disfunción circulatoria observada en la cirrosis hepática con hipertensión portal, con repercusiones sobre el pulmón y la circulación renal. La vasodilatación esplácnica es responsable de la circulación hiperdinámica, que se manifiesta por un descenso de la presión arterial y de la resistencia vascular periférica, aumento del gasto cardiaco y elevación del flujo de sangre al territorio portal. (Catalina-Rodríguez, 2012)

\section{Ascitis}

La ascitis se define como la acumulación patológica de líquido en la cavidad peritoneal. Es la segunda complicación más frecuente de la cirrosis hepática después del hepatocarcinoma, por lo que constituye una forma de descompensación habitual cuyo manejo en la mayoría de los casos puede realizarse de manera ambulatoria. El diagnóstico etiológico de la ascitis se basa en la historia clínica, la ecografía

767 Vol. 5, núm. 3, enero, 2019, pp.759-779

María Fernanda Delgado López, Jonathan Yomar Chamaidan Moreno, Karen Lisseth Labanda Jaramillo, Diana Patricia Delgado Suárez, Diana Gabriela Echeverría Gia, Stefany Gladyz Matute Ortiz 
abdominal y el análisis de líquido ascítico. El desarrollo de ascitis en la historia natural de la cirrosis se asocia a un mal pronóstico, y su aparición constituye una indicación de trasplante hepático.

Su existencia está a menudo asociada con un empeoramiento de la calidad de vida de los enfermos y con la presencia de otras complicaciones como las infecciones bacterianas, la hiponatremia y la insuficiencia renal. Los factores predictivos de mal pronóstico en los pacientes con ascitis son la hiponatremia, la presión arterial baja, la insuficiencia renal y los niveles bajos de excreción de sodio en orina. (P. Bellota, 2012)

\section{Fisiopatología}

La ascitis se debe fundamentalmente a una alteración de la capacidad del riñón de excretar sodio en la orina. En la cirrosis, a medida que progresa la hipertensión portal, se produce una vasodilatación del lecho vascular esplácnico por un incremento de la biodisponibilidad de óxido nítrico (ON), un potente vasodilatador a nivel endotelial. La vasodilatación esplácnica provoca una disminución del volumen arterial efectivo, que es la causa de la activación de los receptores volumétricos arteriales, de los cardiopulmonares y de la activación del sistema simpático y del sistema renina angiotensina aldosterona (SRRA). La retención de sodio provoca una expansión del volumen extracelular que, asociado a la baja presión oncótica plasmática, no permite mantener el agua retenida en el espacio vascular dando lugar a la formación de ascitis y edemas.

En fases muy avanzadas, la vasodilatación esplácnica y el grado de activación de estos sistemas compensadores son muy intensos, apareciendo una marcada vasoconstricción en territorios vasculares no esplácnicos por la acción de la angiotensina II, el sistema adrenérgico y la ADH. Así, los pacientes con cirrosis y ascitis presentan una vasoconstricción renal que es progresivamente más intensa conforme avanza la enfermedad, y que determina una disminución del flujo sanguíneo renal y del filtrado glomerular con la aparición de un fallo renal progresivo denominado síndrome hepatorrenal. (P. Bellota, 2012)

\section{Cuadro clínico}

Este varía de acuerdo a la severidad de la ascitis. Uno de los hallazgos iniciales, es la matidez en flancos, la cual debe ser desplazable con la rotación del paciente a la derecha o a la izquierda. Cuando la ascitis es mayor, podemos encontrar el signo de la "oleada positiva, y cuando ésta es masiva

768 Vol. 5, núm. 3, enero, 2019, pp.759-779

María Fernanda Delgado López, Jonathan Yomar Chamaidan Moreno, Karen Lisseth Labanda Jaramillo, Diana Patricia Delgado Suárez, Diana Gabriela Echeverría Gia, Stefany Gladyz Matute Ortiz 
hallaremos un abdomen con piel lustrosa debido a una ascitis a tensión, acompañada generalmente de dificultad respiratoria, debido a la elevación de los hemidiafragmas. Después del examen del abdomen, el examen físico general puede ayudar a definir la causa de ascitis La presencia de eritema palmar, telangiectasias en los brazos o tórax, hipertrofia parotídea, ginecomastia, circulación colateral abdominal, sugieren que la ascitis es debida a enfermedad hepática. (Vargas, 2007)

\section{Diagnóstico}

- Signos Clínicos

- Ecografía, TAC y Resonancia Magnética abdominales

- Paracentesis diagnóstica: Se debe realizar en todos los pacientes con ascitis de comienzo reciente y todos los pacientes ingresados con ascitis.

\section{Tratamiento}

El $90 \%$ de los pacientes cirróticos con ascitis responden al tratamiento con dieta hiposódica y diuréticos, siendo el de elección la espirinolactona. Antes del comienzo terapéutico debe valorarse el grado de ascitis y descartar la presencia de PBE u otras complicaciones de la cirrosis y debe suspenderse temporalmente ante la presencia de insuficiencia renal o hiponatremia.

- Reposo en cama.

- $\quad$ Restricción de Sal

\section{Terapia Farmacológica}

- Diuréticos de Primera Línea: espirinolactona y furosemida.

- Diuréticos de Segunda Línea: triamtereno e hidroclorotiazida.

- $\quad$ Infusión de Albumina

- Paracentesis

- $\quad$ Shunt peritoneo -venoso:

- Tratamiento de la ascitis refractaria: Se denomina refractaria a la ascitis grado II o III que no responde a tratamiento médico o cuya recidiva precoz no se puede evitar con dicho tratamiento. Aparece en el 5-10\% de los pacientes cirróticos con ascitis. El tratamiento de elección en estos pacientes es la paracentesis evacuadora total de repetición con reposiciónde

769 Vol. 5, núm. 3, enero, 2019, pp.759-779

María Fernanda Delgado López, Jonathan Yomar Chamaidan Moreno, Karen Lisseth Labanda Jaramillo, Diana Patricia Delgado Suárez, Diana Gabriela Echeverría Gia, Stefany Gladyz Matute Ortiz 
albúmina intravenosa y posterior instauración de tratamiento diurético. (Salman Nusrat M. S., Complicaciones de la Cirrosis Hepatica, 2014)

\section{Peritonitis bacteriana espontánea}

La peritonitis bacteriana espontánea se define como la infección del líquido ascítico sin fuente aparente de infección intraabdominal. Se trata de una complicación frecuente y grave de los pacientes con cirrosis hepática avanzada y ascitis. Aproximadamente el 10-30\% de los pacientes cirróticos con ascitis hospitalizados tienen una PBE. El diagnóstico y tratamiento precoz y las medidas preventivas han contribuido a mejorar su pronóstico en los últimos años. (Koulaouzidis A, 2009)

\section{Fisiopatología}

En la cirrosis están alterados los tres mecanismos de defensa fundamentales que en condiciones normales evitan la translocación bacteriana:

- $\quad$ La estabilidad de la flora intestinal

- La integridad del epitelio intestinal y

- $\quad$ Las defensas inmunológicas del huésped.

El mecanismo patogénico clave que inicia la PBE es la translocación bacteriana (TB), proceso por el cual las bacterias entéricas viables y no viables y sus productos cruzan la barrera mucosa intestinal e infectan los ganglios linfáticos mesentéricos, desde donde alcanzan la circulación sanguínea y, posteriormente, el líquido ascítico. Aquellos pacientes con una disminución de la capacidad defensiva del LA han demostrado ser más susceptibles de desarrollar una peritonitis. Esta capacidad de opsonización del LA se correlaciona con los niveles de proteínas totales en el líquido. De hecho, está bien establecido que los pacientes con una disminución de las proteínas totales en el LA tienen mayor riesgo de desarrollar PBE. (González-Alonso R, 2007)

\section{Cuadro clínico}

Generalmente es de curso asintomático y ocasionalmente puede presentar sintomatología tal como: dolor abdominal, signos peritoneales, fiebre. Frecuentemente se presenta de forma inespecífica, como encefalopatía, deterioro de la función renal, sin cuadro clínico de infección abdominal. La aparición de íleo paralítico, hipotensión o hipotermia se produce en estadios avanzados y conlleva un peor pronóstico. Las alteraciones analíticas como la presencia de leucocitosis, la acidosis metabólica o el

770 Vol. 5, núm. 3, enero, 2019, pp.759-779

María Fernanda Delgado López, Jonathan Yomar Chamaidan Moreno, Karen Lisseth Labanda Jaramillo, Diana Patricia Delgado Suárez, Diana Gabriela Echeverría Gia, Stefany Gladyz Matute Ortiz 
deterioro de la función renal obligan siempre a descartar PBE, incluso en ausencia de otras manifestaciones clínicas. (Vargas, 2007)

\section{Diagnóstico}

El diagnóstico de PBE se establece: Paracentesis diagnóstica: Recuento en el LA de polimorfonucleares $\geq 250 / \mu 1$,

\section{Tratamiento}

Ante todo, paciente con diagnóstico de PBE, se debe iniciar tratamiento antibiótico empírico, sin esperar el resultado de los cultivos. El tratamiento antibiótico ideal deberá cumplir los siguientes requisitos: actividad sobre las bacterias más frecuentemente responsables de PBE (enterobacterias y estreptococos), concentraciones adecuadas en líquido ascítico, y ausencia de efectos indeseables sobre la función hepática y renal.

La cefotaxima es el antibiótico que más se ha utilizado. Otras alternativas: ceftriaxona, amoxicilina/ácido clavulánico han demostrado seguridad y eficacia similares a cefotaxima. Debe evitarse el uso de aminoglucósidos debido al riego de nefrotoxicidad y la precipitación de un síndrome hepatorenal. (Vargas, 2007).

\section{Hemorragia por varices gastroesofágicas}

El incremento de la presión portal y los fenómenos de angiogénesis asociados promueven la apertura de colaterales y la consiguiente formación de varices esofagogástricas, que desvían una parte del flujo sanguíneo hacia la vena cava. Globalmente, la probabilidad de que un enfermo con cirrosis hepática desarrolle varices esofágicas es del 5\% al año de establecer el diagnóstico y del 12\% a los tres años. Este hecho no es probable que ocurra sin un gradiente de presión portal superior a $10 \mathrm{~mm} \mathrm{Hg}$. Con el tiempo, el aumento de la presión portal produce una dilatación progresiva de la variz y un adelgazamiento de su pared.

Cuando la presión hidrostática intravaricosa supera el límite elástico de su pared, se genera una tensión excesiva que produce su rotura. El riesgo es más alto entre los pacientes con mala función hepática y varices de gran tamaño o con signos de color rojo (estrías longitudinales o manchas de color rojo cereza sobre la superficie de la variz). Por ejemplo, un paciente con un grado Child C, ascitis a tensión

771 Vol. 5, núm. 3, enero, 2019, pp.759-779

María Fernanda Delgado López, Jonathan Yomar Chamaidan Moreno, Karen Lisseth Labanda Jaramillo, Diana Patricia Delgado Suárez, Diana Gabriela Echeverría Gia, Stefany Gladyz Matute Ortiz 
y varices de gran tamaño con signos endoscópicos de riesgo, presenta una probabilidad de sangrado del 76\%. (Salman Nusrat M. S., 2014)

\section{Manifestaciones clínicas}

Las várices esofágicas no producen dolor ni molestias, excepto cuando sangran. El sangrado por várices es una complicación grave del daño hepático crónico y puede manifestarse de las siguientes maneras:

- Hematemesis

- $\quad$ Melena

- $\quad$ Lipotimia o desmayo

- Anemia crónica, en casos de pequeños sangrados por gastropatía hipertensiva portal. (Villena, 2007)

\section{Diagnóstico}

- $\quad$ Esofagogastroduodenoscopia

- $\quad$ Ecografía con doppler

- Radiografía baritada del esófago y estómago,

- $\quad$ La angiografía de la vena porta y manometría (LaBrecque, 2015).

\section{Tratamiento}

- Valoración y reanimación hemodinámica:

- Valoración de tensión arterial, frecuencia cardiaca, diuresis y signos externos de hipoperfusión tisular.

- Colocación de 2 vías venosas de grueso calibre a la vez que se extrae sangre para hemograma, bioquímica, coagulación y pruebas cruzadas.

- Infusión de expansores plasmáticos.

- Valoración de transfusión de concentrados de hematíes, plasma fresco congelado y/o concentrados de plaquetas.

- Tratamiento Hemostático farmacológico:

- El tratamiento con los fármacos vasoactivos debe iniciarse ante la sospecha del sangrado por varices, antes incluso del diagnóstico endoscópico.

772 Vol. 5, núm. 3, enero, 2019, pp.759-779

María Fernanda Delgado López, Jonathan Yomar Chamaidan Moreno, Karen Lisseth Labanda Jaramillo, Diana Patricia Delgado Suárez, Diana Gabriela Echeverría Gia, Stefany Gladyz Matute Ortiz 
Estudio de casos sobre la cirrosis hepática y sus complicaciones en el Hospital Universitario de

- Somatostatina

- Octeotrido

- Terlipresina / Glipresina

- Tratamiento hemostático endoscópico:

- Taponamiento esofágico:

- Derivación porto-sistémica intrahepáticatransyugular

(DPPI)

o

“Transjugularintrahepaticportosystemic shunt” (TIPS )

\section{Análisis y discusión de los resultados}

Este estudio se realizó en el periodo enero 2015 hasta diciembre del 2015

\section{Recursos empleados}

- Talento Humano

- Investigadora - Tutor

\section{Recursos físicos}

- Insumos de oficina

- Datos estadísticos de la Entidad de salud: Se asumió el modelo de investigación descriptiva, documental de campo, empleando las técnicas de la observación, el estudio de casos y el análisis de contenido en este caso de las historias clínicas de los pacientes de este hospital.

\begin{tabular}{crr}
\hline Complicaciones & Emergencia del HUG & \% \\
\hline Ascitis & 85 & $31 \%$ \\
Hemorragia digestiva & 172 & $63 \%$ \\
Síndrome Hepatorenal & 12 & $4 \%$ \\
Varices esofágicas & 6 & $\mathbf{2 \%}$ \\
\hline TOTAL & $\mathbf{2 7 5}$ & $\mathbf{1 0 0 \%}$
\end{tabular}

Fuente: Datos tomados de las Historias Clínicas en pacientes entre 55 y 65 años de edad

Periodo 2015. Elaboración: propia (2019)

773 Vol. 5, núm. 3, enero, 2019, pp.759-779

María Fernanda Delgado López, Jonathan Yomar Chamaidan Moreno, Karen Lisseth Labanda Jaramillo, Diana Patricia Delgado Suárez, Diana Gabriela Echeverría Gia, Stefany Gladyz Matute Ortiz 
Dom. Cien., ISSN: 2477-8818

Vol. 5, núm. 3, enero, 2019, pp. 759-779

Estudio de casos sobre la cirrosis hepática y sus complicaciones en el Hospital Universitario de

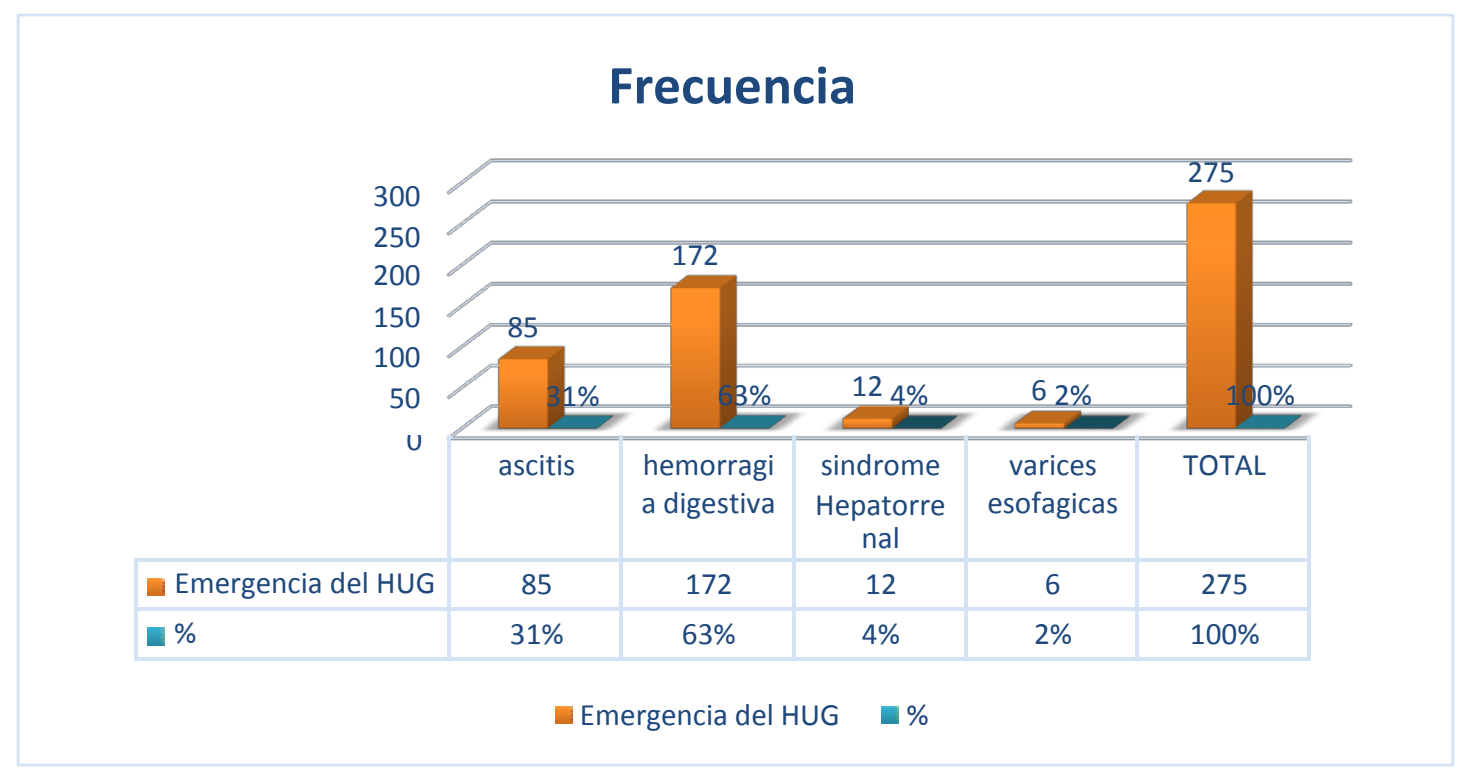

Fuente Departamento de estadística del Hospital Universitario de Guayaquil

Elaboración: Delgado (2019)

\section{Análisis}

En este grafico podemos observar que la cantidad de pacientes entre 55 y 65 años de edad en los datos obtenidos de las Historias Clínicas del Hospital Universitario de Guayaquil con complicaciones de la Cirrosis Hepática ha atendido la ocurrencia de 275 casos en el transcurso del año 2015. Observamos que, dentro de las complicaciones, la más frecuente es la Hemorragia Digestiva con un porcentaje de $63 \%$ del total de datos.

\begin{tabular}{lrrrr}
\hline COMPLICACIONES & \multicolumn{5}{c}{ SEXO } \\
\hline & MASCULINO & \% MASCULINO & FEMENINO & $\%$ FEMENINO \\
\hline ASCITIS & 33 & $21,71 \%$ & 52 & $42,3 \%$ \\
HEMORRAGIA DIGESTIVA & 105 & $69,08 \%$ & 67 & $54,47 \%$ \\
SINDROME HEPATORRENAL & 8 & $5,26 \%$ & 4 & $3,25 \%$ \\
VARICES ESOFAGICAS & 6 & $3,95 \%$ & & \\
\hline \multicolumn{1}{c}{ Total } & 152 & $100 \%$ & 123 & $100 \%$ \\
\hline
\end{tabular}

Fuente: Datos tomados de las Historias Clínicas en pacientes entre 55 y 65 años de edad

Periodo 2015. Elaboración: propia (2019)

774 Vol. 5, núm. 3, enero, 2019, pp.759-779

María Fernanda Delgado López, Jonathan Yomar Chamaidan Moreno, Karen Lisseth Labanda Jaramillo, Diana Patricia Delgado Suárez, Diana Gabriela Echeverría Gia, Stefany Gladyz Matute Ortiz 
Estudio de casos sobre la cirrosis hepática y sus complicaciones en el Hospital Universitario de Guavaquil, año 2015.

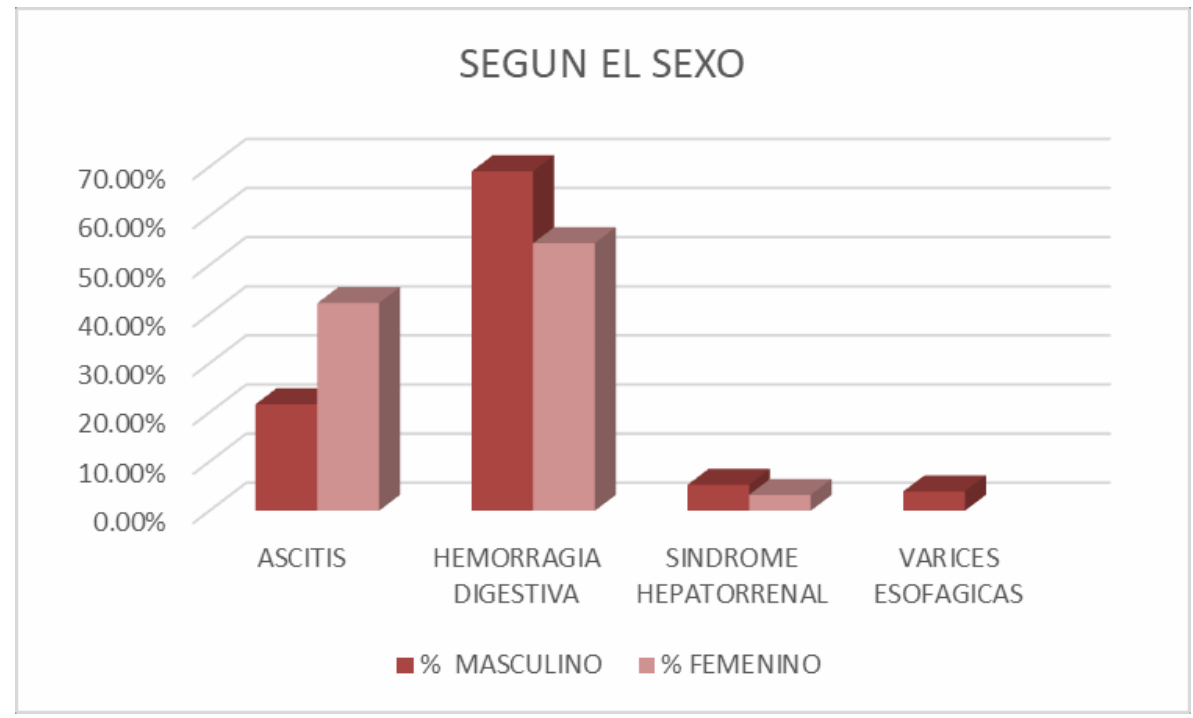

Fuente Datos del departamento de Estadística del Hospital Universitario de Guayaquil Elaboración Delgado (2019)

\section{Análisis}

Según lo que refleja esta tabla la complicación más frecuente es la Hemorragia Digestiva en el sexo masculino, la ascitis nos refleja que es más frecuente en el sexo femenino, el síndrome hepatorenal es más frecuente en el sexo masculino, y varices esofágicas con frecuencia en sexo masculino sin presentar rango dentro del sexo femenino. Esto nos quiere decir que la hemorragia digestiva es la complicación más frecuente dentro del rango de 55 a 65 años de edad.

\begin{tabular}{lcc}
\hline \multicolumn{3}{l}{ COMPLICACIONES SEGÚN EL RANGO DE EDAD } \\
\hline EDAD & FRECUENCIA & $\%$ \\
$55-59$ & 99 & $36 \%$ \\
$60-65$ & 176 & $64 \%$ \\
\hline TOTAL & 275 & $100 \%$ \\
\hline
\end{tabular}

Fuente: Datos tomados de las Historias Clínicas en pacientes entre 55 y 65 años de edad

Periodo 2015. Elaboración propia (2019)

775 Vol. 5, núm. 3, enero, 2019, pp.759-779

María Fernanda Delgado López, Jonathan Yomar Chamaidan Moreno, Karen Lisseth Labanda Jaramillo, Diana Patricia Delgado Suárez, Diana Gabriela Echeverría Gia, Stefany Gladyz Matute Ortiz 


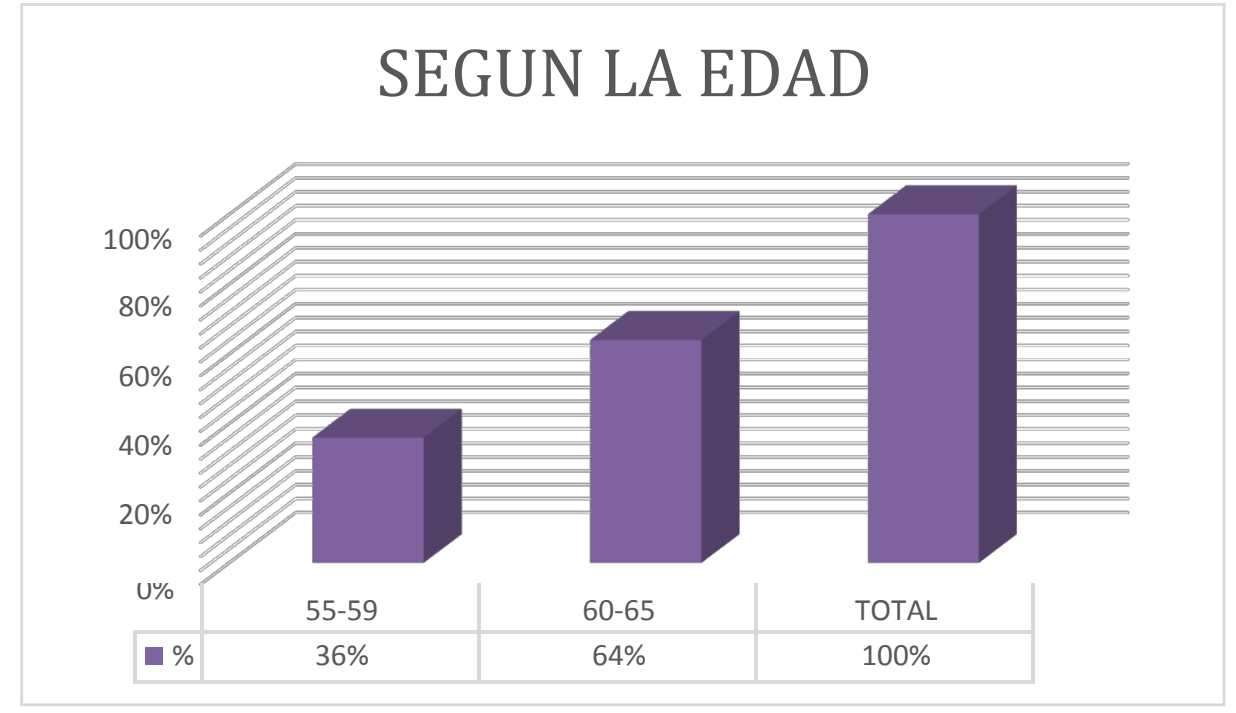

Fuente: Datos proporcionados por el Departamento de Estadística del Hospital Universitario de Guayaquil

Elaboración: Delgado (2019)

\section{Análisis}

En este grafico se puede observar que durante el año 2015 la más alta incidencia de casos se presentó en el rango de edad de 60-65 años de edad con un porcentaje de $64 \%$, y con un porcentaje menor $36 \%$ en el rango de edad de 55-59 años de edad.

\begin{tabular}{|c|c|c|c|c|c|}
\hline \multicolumn{6}{|c|}{ SEGÚN EL NIVEL SOCIOECOMICO } \\
\hline EDAD & ALTO & MEDIO & $\%$ MEDIO & BAJO & $\%$ BAJO \\
\hline $55-59$ & 0 & 50 & $43 \%$ & 58 & $36 \%$ \\
\hline $60-65$ & 0 & 65 & $57 \%$ & 102 & $64 \%$ \\
\hline TOTAL & & 115 & 1 & 160 & 1 \\
\hline
\end{tabular}

Fuente: Datos tomados de las Historias Clínicas en pacientes entre 55 y 65 años de edad

Periodo 2015. Elaboración propia (2019)

776 Vol. 5, núm. 3, enero, 2019, pp.759-779

María Fernanda Delgado López, Jonathan Yomar Chamaidan Moreno, Karen Lisseth Labanda Jaramillo, Diana Patricia Delgado Suárez, Diana Gabriela Echeverría Gia, Stefany Gladyz Matute Ortiz 
Estudio de casos sobre la cirrosis hepática y sus complicaciones en el Hospital Universitario de

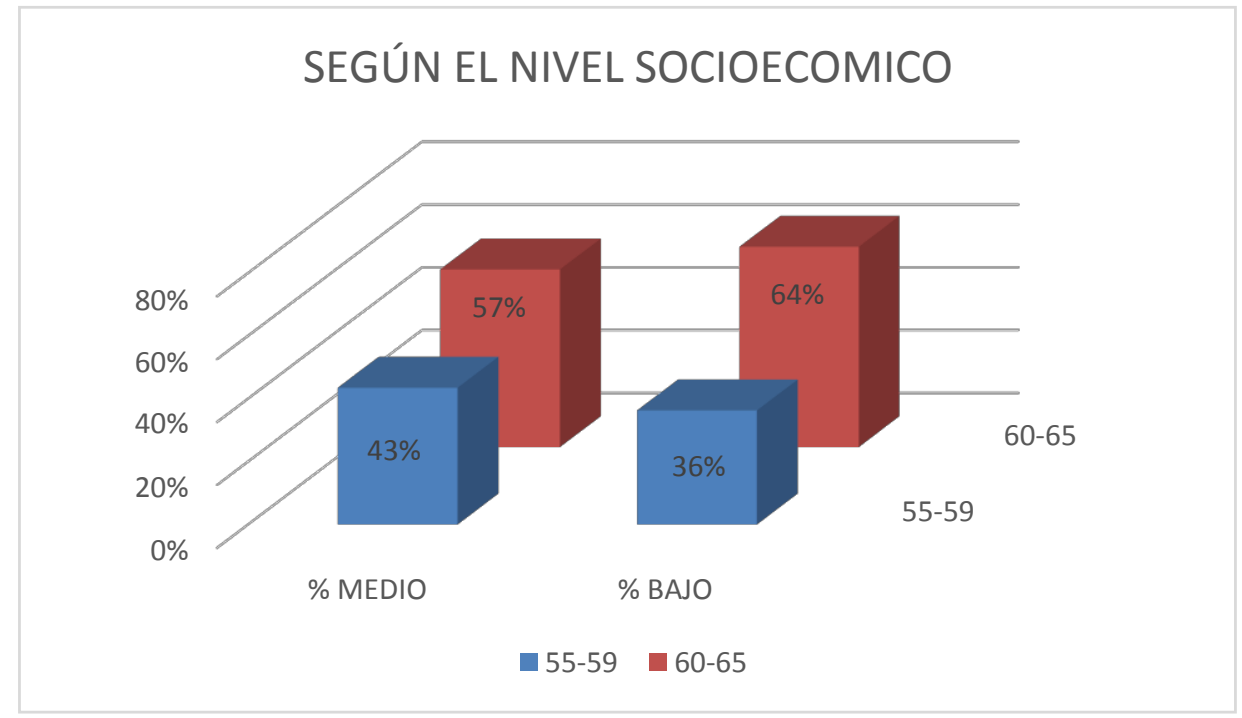

Fuente: Datos aportados por el Hospital Universitario de Guayaquil

Elaboración: Delgado (2019)

Análisis

Realizamos un cuadro comparativo relacionando la edad con el nivel socioeconómico, por medio de rango. Aproximadamente un $64 \%$ corresponde a pacientes con bajo nivel socioeconómico y edad de 60-65 años de edad.

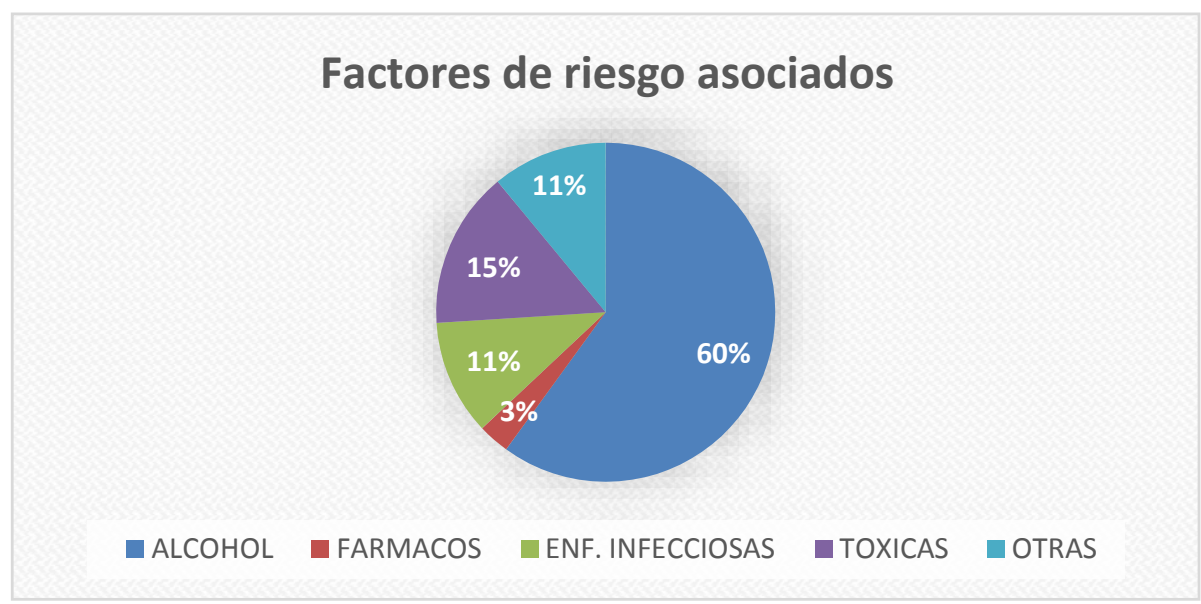

Fuente datos proporcionados por el Hospital Universitario de Guayaquil

Elaboración: Delgado (2019)

\section{Análisis}

De los 275 pacientes empleados como muestra en esta imagen ilustrativa nos refleja que aproximadamente un $60 \%$ de los pacientes que presentas complicaciones de la Cirrosis Hepática son

777 Vol. 5, núm. 3, enero, 2019, pp.759-779

María Fernanda Delgado López, Jonathan Yomar Chamaidan Moreno, Karen Lisseth Labanda Jaramillo, Diana Patricia Delgado Suárez, Diana Gabriela Echeverría Gia, Stefany Gladyz Matute Ortiz 
dependientes activos del alcohol con un porcentaje de $60 \%$, sustancias toxicas con un $15 \%$, enfermedades infecciosas con un $11 \%$, fármacos $3 \%$, entre otras con un $11 \%$.

\section{Conclusiones}

El estudio de investigación se centró, en las complicaciones que se presentan en el transcurso de la Cirrosis Hepática en los pacientes de la emergencia del Hospital Universitario de Guayaquil, para llevar a cabo el estudio se tomó el estudio de 275 pacientes, a continuación, analizaremos cada uno de los resultados.

Como conclusión los pacientes que acuden con mayor frecuencia al Hospital Universitario dentro del periodo antes mencionado y con la complicaciones especificas son: Ascitis con un porcentaje del 31 \%, Hemorragia Digestiva 63\%, Síndrome Hepatorrenal 4\% y varices esofágicas con un 2\% del total tomados de los 275 pacientes que acudieron en el transcurso del año.

En relación al sexo de acuerdo a cada una de las complicaciones nos dio como resultado un dato interesante que las complicaciones que más afecta al género femenino es la Ascitis con un porcentaje del 42,3\% en comparación con el género masculino que solo representa un $21,7 \%$. E incluso durante el estudio pudimos concluir que las mujeres acuden con mayor frecuencia con ascitis refractaria.

En relación a la edad tomamos como referencias dos escalas de rango de edad desde 55 hasta 59 años de edad y desde 60 hasta 65 años de edad el cual nos dio como resultado que la edad más susceptible a las complicaciones es de 60 hasta 65 años de edad con un porcentaje del $64 \%$.

Las factores asociados o factores de riesgo el que más sobresale de toda la investigación dentro de los hábitos y encuesta social es el alcoholismo en hombres con un porcentaje del $60 \%$, pero que en la actualidad la mujer también ha tomado un lugar importante dentro de este factor, en segundo lugar y con un porcentaje del $15 \%$ tóxicos, $11 \%$ enfermedades infecciosas.

\section{Referencias Bibliográficas}

AYALA, A.-E. G. (2012). Cirrosis hepática. Farmacia abierta, 45.

Buey, L. G. (2012). Cirrosis Hepatica. Medicine, 11(11):625-33.

Catalina-Rodríguez, D. R.-R.-C. (2012). Hipertensión Portal. Medicine, 11(11):634-43.

D’Amico G, G.-T. G. (2006). Natural history and prognostic indicators of survival in cirrhosis: a systematic review of 118 studies. Medicine, 44:217-31.

778 Vol. 5, núm. 3, enero, 2019, pp.759-779

María Fernanda Delgado López, Jonathan Yomar Chamaidan Moreno, Karen Lisseth Labanda Jaramillo, Diana Patricia Delgado Suárez, Diana Gabriela Echeverría Gia, Stefany Gladyz Matute Ortiz 
Elvira Martínez Leyva, V. H. (2006). Síndrome Hepatopulmonar. Medigraphic, 51 (3): 124-133.

FARFAN G, C. C. (2002). Mortalidad de Enfermedades Hepatobiliares. Rev. Gastroenterológica de Perú, 22(4):212-233.

González-Alonso R, G.-G. M.-M. (2007). Fisiopatología de la translocación y la peritonitis bacteriana espontánea en la cirrosis. Gastroenterol Hepato. Scielo, 30: 78-84.

LaBrecque, P. D. (2015). Guías mundiales de la Organización Mundial de Gastroenterología. World Gastroenterology Organisation, 2013, 5.

MsC. Raúl Toranzo Labrada, M. R. (2012). Síndrome Hepatorrenal: diagnóstico y tratamiento. Medisan, 16(5): 786.

P. Bellota, b. B.-M. (2012). Ascitis y síndrome hepatorenal. Medicine, 11(11):644-51.

R., D. f. (2009). Evolving consensus in portal hypeetension. 43:167-76.

Salman Nusrat, M. S. (2014). Cirrhosis and its complications: Evidence based treatment. World J Gastroenterol , 20(18): 5442-5460.

Sergio Muñoz C., A. B. (2006). Síndrome Hepatopulmonar. Revista HCUCh, 17: 229 - 37.

Vanesa Bernal, J. B. (2003). Cirrosis Hepatica. *Unidad de Hepatología. Instituto de Enfermedades Digestivas y Metabólicas IDIBAPS. CIBERehd, 595-624.

Vargas, R. R. (2007). ASCITIS. ESSALUD, 24(1)35.

779 Vol. 5, núm. 3, enero, 2019, pp.759-779 María Fernanda Delgado López, Jonathan Yomar Chamaidan Moreno, Karen Lisseth Labanda Jaramillo, Diana Patricia Delgado Suárez, Diana Gabriela Echeverría Gia, Stefany Gladyz Matute Ortiz 